\title{
ESTUDO DO COMPORTAMENTO DINÂMICO DE BLINDAGEM ONDULADA EM PROTEÇÃO BALÍSTICA
}

\author{
Daniel Fonseca da Cunha' \\ Achille Arantes Bassi ' \\ Rodrigo Felix de Araujo Cardoso ${ }^{2}$ \\ Luiz Paulo Mendonça Brandão ' \\ Alaelson Vieira Gomes '
}

\section{Resumo}

Aliar proteção balística e mobilidade é um grande desafio quando se utiliza materiais metálicos. Pensando em suprir essa necessidade, neste trabalho é analisada a blindagem metálica ondulada. Esse tipo de blindagem consiste em chapas metálicas com superfícies onduladas, que criam superfícies côncavas e convexas ao longo da chapa, podendo assim melhorar - desempenho em impactos balísticos. Para comprovar a eficácia desta blindagem foram realizados ensaios balísticos e análises computacionais por meio do software AUTODYN ${ }^{\circledR}$, que utiliza o método de elementos finitos. Os resultados das simulações mostram que para um mesmo volume de material, a blindagem ondulada é capaz de prover maior proteção às perfurações balísticas do que superfícies planas, o que foi corroborado pelos ensaios balísticos. Desse modo, esse tipo de blindagem apresenta potencial para se tornar uma alternativa viável para proteção balística em veículos leves. Palavras-chave: Comportamento dinâmico; Blindagem balística; Simulação computacional; Ensaio balístico.

\section{STUDY OF DYNAMIC BEHAVIOR OF WAVY ARMOR IN BALLISTIC PROTECTION}

\begin{abstract}
Combining ballistic protection and mobility is a huge challenge when using metallic materials. Thinking about supplying this need, the wavy metallic armor is analyzed in this work. This type of armor consists of metal sheet with concave and convex surfaces along the plate, improving performance on ballistic impacts. To prove the effectiveness of this armor, ballistic tests and computational analyzes were performed through AUTODYN ${ }^{\circledR}$ software, which uses the finite element method. The results of the simulations show that for the same volume of material, the wave armor is able to provide greater protection to ballistic perforations than flat surfaces, which was corroborated by ballistic tests. Therefore, that type of armor has potential to become a viable alternative for ballistic protection in light vehicles.
\end{abstract}

Keywords: Dynamic behavior; Ballistic armor; Computational simulation; Ballistic tests.

\section{INTRODUÇÃO}

O avanço tecnológico aplicado à indústria bélica torna imprescindível os estudos e o desenvolvimento de novos meios de prover proteção balística às unidades móveis. Tais estudos podem otimizar materiais e métodos de blindagem a fim de se aumentar a segurança e desenvolver novos materiais para essa aplicação. Desse modo, este trabalho se propõe estudar a blindagem metálica ondulada.

A blindagem metálica ondulada consiste em chapas metálicas com superfícies onduladas, com formas côncavas e convexas alternadas ao longo da chapa. Segundo Gomes [I], a geometria convexa da superfície de materiais cerâmicos pode melhorar resultados para impactos balísticos quando comparados às placas cerâmicas planas, o que indica que essa geometria permite a possibilidade de se usar chapas mais finas e consequentemente com menor densidade superficial, além de alterar a trajetória do projétil, retirando-o de uma incidência perpendicular à superfície do alvo [2].

A blindagem proposta visa se equiparar ao nível III, segundo a norma NBR I 5000. Tal nível de proteção é capaz de prover segurança até disparos de projéteis $7.62 \times 5$ IFMJ

'Seção de Engenharia Mecânica e de Materiais, Instituto Militar de Engenharia - IME, Rio de Janeiro, RJ, Brasil. E-mail: danielfonsecacunha@gmail.com ${ }^{2}$ Seção de Tecnologia Mecânica, Centro Brasileiro de Pesquisas Físicas - CBPF, Rio de Janeiro, RJ, Brasil.

2 I 76-I523 (C) 2020. Cunha et al. Publicado pela ABM. Este é um artigo publicado em acesso aberto (Open Access) sob a licença Creative Commons Attribution, que permite uso, distribuição e reprodução em qualquer meio, sem restrições desde que o trabalho original seja corretamente citado. 
(Full Metal Jacket) [3]. Para avaliar a eficácia da geometria, foi realizada uma análise qualitativa computacional e testes balísticos.

\section{MATERIAIS E MÉTODOS}

Os aços maraging possuem limite de resistência e tenacidade à fratura superiores aos aços convencionais de alta resistência e, portanto, foi escolhido o maraging 250 para se avaliar a geometria no ensaio balístico. Entretanto, devido à falta de parâmetros experimentais disponíveis acerca deste material capazes de modelar o fenômeno desejado por meio de modelos constitutivos, foi utilizado o aço ABNT/ AISI 4340. Material amplamente utilizado em blindagens balísticas e capaz de fornecer uma resposta qualitativa a respeito da variação de geometria das superfícies de impacto.

Por se tratar de um evento que envolve certa complexidade, altas velocidades, altas taxas de deformação, amolecimento local e até mesmo desintegração do projétil durante o impacto [4], o impacto do projétil foi modelado computacionalmente por meio do método de elementos finitos onde o projétil 7.62X5 I FMJ (Full Metal Jacket) possui núcleo de chumbo e jaqueta de latão, com $9,78 \mathrm{~g}$ de massa e possui velocidade de $850 \mathrm{~m} / \mathrm{s}$ e velocidade angular em torno do seu eixo de $17.522 \mathrm{rad} / \mathrm{s}$.

Nas simulações em superfícies convexas, foram utilizadas placas baseadas em uma função senoidal do tipo $F(X)=15 \operatorname{sen}(\pi X / 50)$, com comprimento da placa equivalente a um período da função ( $100 \mathrm{~mm}$ ) e espessura máxima de $3 \mathrm{~mm}$. Tal geometria foi escolhida por possuir o mesmo volume de uma placa plana com largura e comprimento similares, possuindo em seu ponto de maior espessura, a mesma espessura da placa plana.

A avaliação das superfícies foi feita por meio de análises computacionais realizadas por meio do emprego do software AUTODYN ${ }^{\circledR}$, sendo os parâmetros utilizados

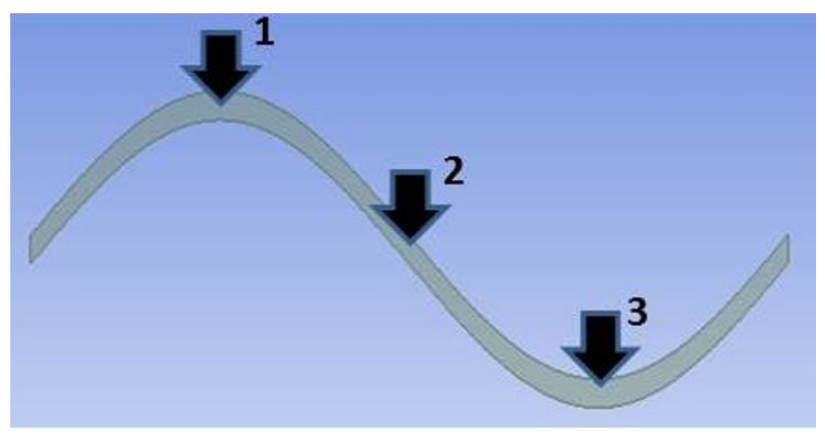

Figura I. Posições de impacto balístico nas simulações computacionais. provenientes do software. Nessa simulação, foi fixado que os projéteis atingiriam a placa ondulada em posições equivalentes a I/4, I/2 e 3/4 do comprimento da placa como ilustra a Figura le, no caso da placa plana, no centro dela. Em todas as análises foi desprezado o efeito de borda no resultado obtido.

A equação constitutiva modelada por Johnson-Cook (Equação I) é utilizada para descrever o comportamento das placas de aço e da jaqueta de latão. Tal equação representa a influência da temperatura no comportamento do material nesse tipo de evento dinâmico [5]:

$$
\sigma=\left[A+B \varepsilon_{p}^{n}\right]\left[1+C \ln \varepsilon_{p}^{*}\right]\left[1-\left(T_{H}\right)^{m}\right]
$$

em que $A$ é a tensão de escoamento inicial, $B$ é a constante de encruamento, $\varepsilon_{\mathrm{p}}$ é a deformação plástica equivalente, $\mathrm{n}$ é o expoente de encruamento, $C$ é a constante da taxa de deformação, $m$ é o expoente de amolecimento térmico, $\dot{\varepsilon}_{\mathrm{p}}^{*}$ é a taxa de deformação plástica e $\mathrm{T}_{\mathrm{H}}$ é a temperatura homóloga, sendo dependente de $T_{f}$, temperatura de fusão, e de $\mathrm{T}_{\mathrm{amb}}$, temperatura do ambiente (Equação 2). Os valores das constantes utilizadas na modelagem estão apresentados na Tabela I.

$$
\mathrm{T}_{\mathrm{H}}=\frac{\left(\mathrm{T}-\mathrm{T}_{\mathrm{amb}}\right)}{\left(\mathrm{T}_{\mathrm{f}}-\mathrm{T}_{\mathrm{amb}}\right)}
$$

Para descrever o comportamento do núcleo de chumbo foi utilizado o modelo de resistência de Steinberg-Guinan (Equação 3 e Equação 4) [6]:

$$
\begin{aligned}
& Y=Y_{0}\left[1+\left(\frac{Y_{P}^{\prime}}{Y_{0}}\right) \cdot\left(\frac{P}{\eta^{1 / 3}}\right)+\left(\frac{G_{T}^{\prime}}{G_{0}}\right)(T-300)\right](1+\beta \varepsilon)^{n} \\
& \mathrm{G}=\mathrm{G}_{0}\left[1+\left(\frac{\mathrm{G}_{\mathrm{P}}^{\prime}}{\mathrm{G}_{0}}\right) \cdot\left(\frac{\mathrm{P}}{\eta^{1 / 3}}\right)+\left(\frac{\mathrm{G}_{\mathrm{T}}^{\prime}}{\mathrm{G}_{0}}\right)(\mathrm{T}-300)\right]
\end{aligned}
$$

em que $Y$ é a tensão de escoamento (yield strength), $Y_{0}$ é a tensão de escoamento inicial, $Y^{\prime}$ ' é a derivada $d Y / d P$ ', $P$ é a pressão, $\eta$ é a compressão definida pela razão do volume específico inicial e o volume específico, $\mathrm{G}_{\mathrm{T}}$ é a derivada $\mathrm{dG} / \mathrm{dT}$ '. G é o modo de cisalhamento, $\mathrm{G}_{0}$ é o módulo de cisalhamento inicial, T a temperatura, $\beta$ é o coeficiente de encruamento, $\varepsilon$ é a deformação plástica e n é o expoente de encruamento. As constantes utilizadas estão apresentadas na Tabela 2.

Para o aço ABNT/AISI 4340 foi adotado o critério de falha de Johnson-Cook, o qual possui uma função de dano cumulativo (Equação 5), sendo a erosão determinada a partir do critério de falha:

Tabela I. Valores das constantes utilizadas para o aço e o latão

\begin{tabular}{cccccccc}
\hline Material & $\mathbf{A}(\mathbf{k P a})$ & $\mathbf{B}(\mathbf{k P a})$ & $\mathbf{n}$ & $\mathbf{C}$ & $\mathbf{m}$ & $\mathbf{T}_{\mathbf{0}} \mathbf{( K )}$ & $\mathbf{T}_{\mathbf{f}}(\mathbf{K})$ \\
\hline Aço & $7,92 \mathrm{e}+05$ & $5,10 \mathrm{e}+05$ & 0,26 & 0,014 & $\mathrm{I}, 03$ & 295,15 & 1793 \\
Latão & $\mathrm{I}, 12 \mathrm{e}+05$ & $5,05 \mathrm{e}+0{ }^{\prime} 5$ & 0,42 & 0,009 & $\mathrm{I}, 68$ & 295,15 & 1189 \\
\hline
\end{tabular}

$\mathrm{n}$ : expoente de encruamento; C: constante da taxa de deformação; m: expoente de amolecimento térmico. 


$$
D=\Sigma\left(\frac{\Delta \varepsilon_{p}}{\varepsilon^{f}}\right)
$$

Em que $\Delta \varepsilon_{\mathrm{p}}$ é o incremento da deformação plástica equivalente e $\varepsilon$ é a deformação de falha. A falha é detectada quando a variável de dano $D$ (parâmetro de dano) atinge o valor unitário, sendo a função deformação de falha (Equação 6) definida por:

$$
\mathrm{E}^{f}=\left[D_{1}+D_{2} \cdot e^{D_{3} \sigma^{*}}\right] \cdot\left[1+D_{4} \cdot \ln |\dot{\varepsilon} *|\right] \cdot\left[1+D_{5} \cdot T_{H}\right]
$$

Em que $D_{n}, D_{2}, D_{3}, D_{4}, D_{5}$ são as constantes de dano, $\sigma^{*}$ é a razão entre a pressão e a tensão de escoamento, $\varepsilon^{*}$ é a taxa de deformação plástica e $\mathrm{T}_{\mathrm{H}}$ é a temperatura homóloga. As constantes utilizadas na modelagem estão apresentadas na Tabela 3.

Foi utilizada também uma Equação de Estado (EOS - Equation of State) linear para o aço, baseada no módulo de elasticidade volumétrica (bulk modulus). Para o chumbo e o latão, a Equação de Estado (EOS) de Choque Linear foi utilizada, em que foram considerados os parâmetros apresentados na Tabela 4.

Para os testes balísticos foram utilizadas placas de aço maraging 250 conforme AMS 65 I 2-H [7] e MIL-S-46850D [8] com dimensões aproximadas de $200 \mathrm{~mm} \times 200 \mathrm{~mm} \times 3 \mathrm{~mm}$ e superfície ondulada, cedidos pela empresa Villares Metals S.A.. Nos ensaios balísticos foram utilizados 2 conjuntos de placas onduladas e 2 conjuntos de placas planas. Os conjuntos contaram com 2 placas de aço e I placa de alumínio, conforme a Figura 2.

As placas de aço foram mantidas juntas através de solda em suas extremidades, enquanto a separação com a placa de alumínio foi feita através de uma camada de epóxi nas extremidades das placas.

Para avaliar o desempenho balístico, foram efetuados 4 disparos em posições próximas aos pontos observados na Figura 2, sendo todos os disparos efetuados com projéteis M80 FMJ.

\begin{tabular}{|c|c|c|c|c|c|c|}
\hline $\mathbf{Y}_{0}(\mathrm{KPa})$ & $\mathbf{Y}_{\mathrm{P}}^{\prime}$ & $\mathbf{G}_{T}^{\prime}$ & $\mathbf{G}_{0}(\mathrm{KPa})$ & $\beta$ & $\mathbf{n}$ & $\mathbf{G}_{\mathrm{P}}$ \\
\hline $8,0 e+3$ & $9.304 \mathrm{e}-04$ & $-9,976 e+03$ & $8,6 e+06$ & 110 & 0,52 & 1,0 \\
\hline
\end{tabular}

Tabela 2. Valores das constantes utilizadas para o chumbo

Tabela 3. Valores das constantes de dano

\begin{tabular}{ccccc}
\hline $\mathbf{D}_{1}$ & $\mathbf{D}_{2}$ & $\mathbf{D}_{3}$ & $\mathbf{D}_{4}$ & $\mathbf{D}_{5}$ \\
\hline $\mathbf{0 , 0 5}$ & 3,44 & $-2,12$ & 0,002 & 0,61 \\
\hline
\end{tabular}
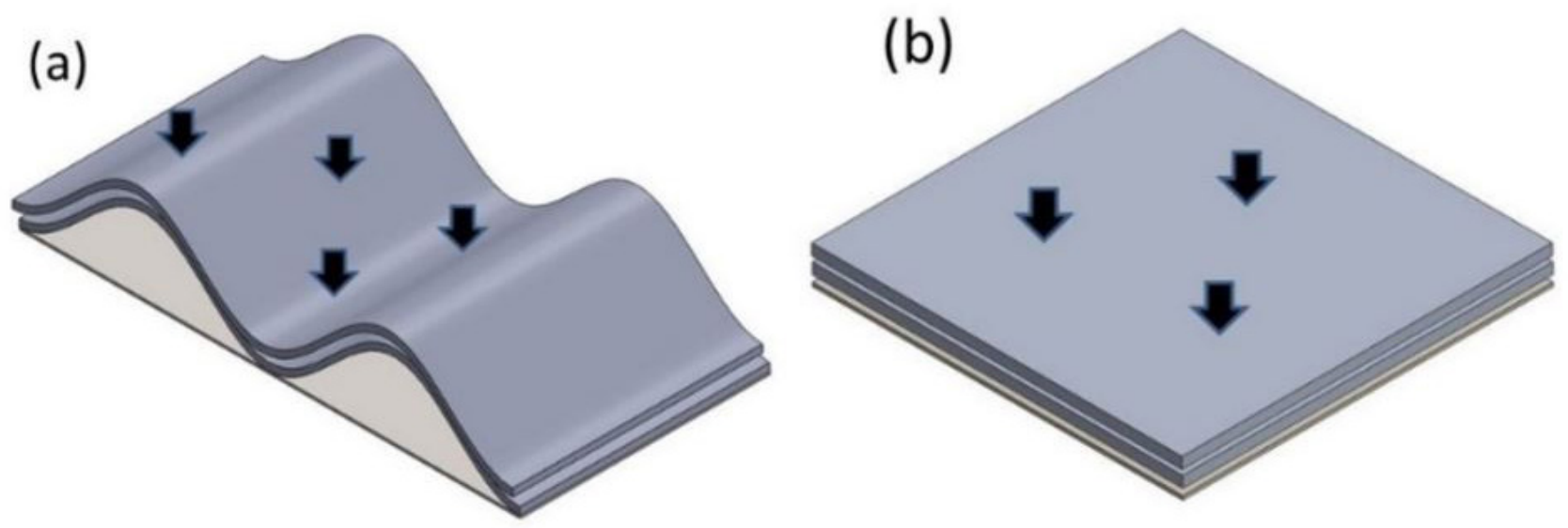

Figura 2. Pontos de impacto balístico em conjuntos de placas onduladas (a) e planas (b).

Tabela 4. Parâmetros da EOS para o chumbo e o latão

\begin{tabular}{lcc}
\hline \multicolumn{1}{c}{ Parâmetro } & Chumbo & Latão \\
\hline Coeficiente de Gruneisen & 2,74 & 2,04 \\
Parâmetro $C_{0}(\mathrm{~m} / \mathrm{s})$ & $2,006 \mathrm{e}+03$ & $3,726 \mathrm{e}+03$ \\
Parâmetro S & 1,429 & 1,434 \\
Temperatura de referência $(\mathrm{K})$ & 295,15 & 295,15 \\
Calor específico $(\mathrm{J} / \mathrm{kgK})$ & 124 & 385 \\
\hline
\end{tabular}

Tecnol. Metal. Mater. Miner., São Paulo, 


\section{RESULTADOS}

$\mathrm{Na}$ Tabela 5 são apresentados os resultados numéricos de velocidade residual do projétil, isto é, após atravessar o alvo, bem como a energia do projétil que foi absorvida pelo material da blindagem nas configurações de blindagem plana e ondulada, neste caso, nos pontos I a 3 (Figura 2). Os valores numéricos foram obtidos com base no elemento apresentado em vermelho (Figura 3).

A Figura 4 apresenta os resultados dos conjuntos das placas ondulares (I, II) e placas planas (III e IV) após o ensaio balístico. A ordem alfabética apresentada em cada

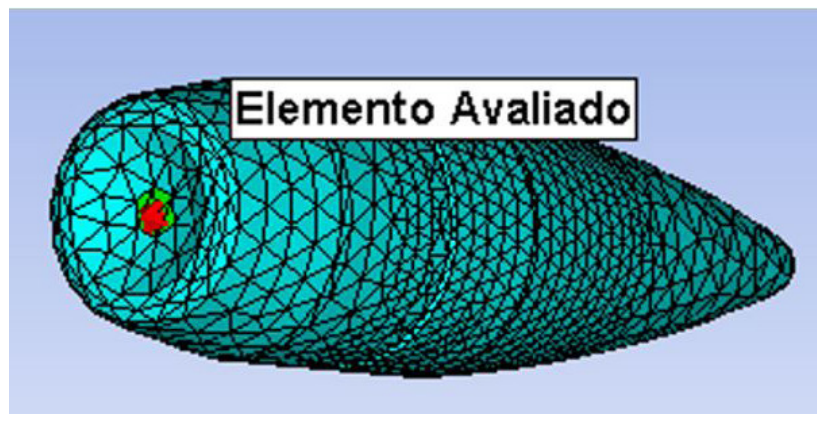

Figura 3. Representação da malha do projétil.
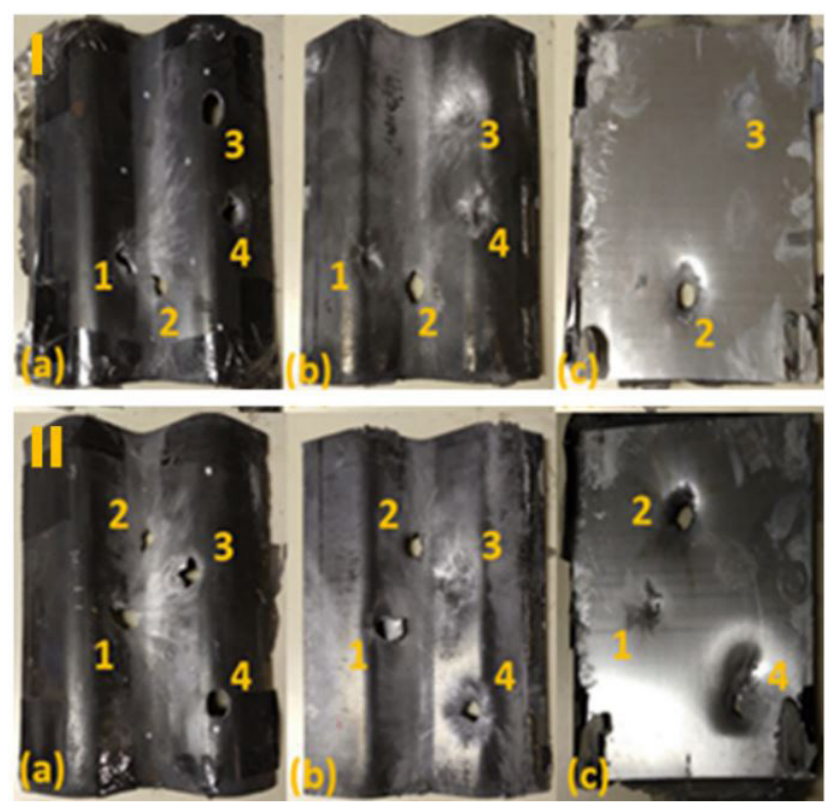

placa indica a ordem de sobreposição entre elas, conforme ilustrado na Figura 2. Nas imagens também estão indicadas as posições de impacto de cada projétil na ordem em que foram disparados (I, 2, 3 e 4).

\section{DISCUSSÃO}

De acordo com os resultados obtidos nas simulações, pode-se dizer que a superfície ondulada obteve melhores resultados a impactos balísticos em todas as posições, porém apresentou pior desempenho na posição 3 , apresentando comportamento próximo ao de uma placa plana no que se refere a velocidade residual. No entanto, na posição 3 houve uma absorção de energia maior por parte da placa do que a observada na posição 2 . Isso nos indica que, embora a velocidade residual não tenha sofrido uma queda significativa, o projétil perdeu mais energia nessa posição, sugerindo que este sofreu maior dano ao atravessar o alvo.

Outro fato relevante observado durante as simulações, foi a deflexão do projétil no impacto na posição 2 , indicando a possibilidade de desvio do projétil no teste real.

Após o ensaio balístico, a partir da observação das perfurações, fica evidente que a configuração ondulada de
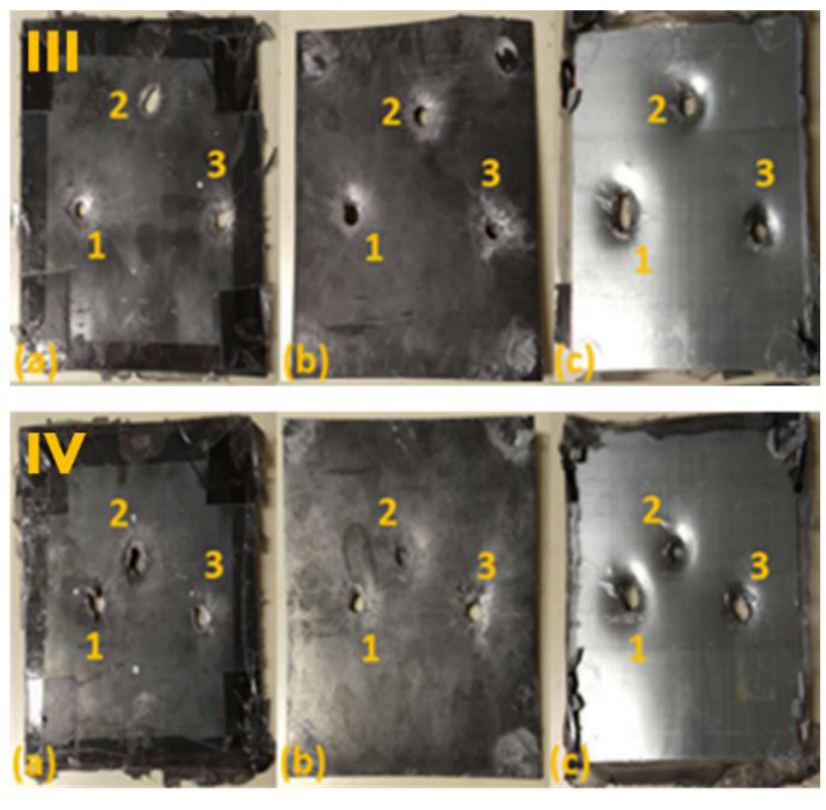

Figura 4. Conjuntos de placas ondulares (I e II) e de placas planas (III e IV) utilizados no ensaio balístico. As letras (a), (b) e (c) indicam a sequência de sobreposição das placas durante o ensaio.

Tabela 5. Resultados numéricos obtidos via modelagem computacional

\begin{tabular}{cccc}
\hline & & Velocidade residual (m/s) & Energia absorvida pela placa (J) \\
\hline Plana & & 758,2 & 620,53 \\
\hline \multirow{2}{*}{ Ondulada } & Posição I & 670,55 & 844,52 \\
& Posição 2 & 695,98 & 627,33 \\
& Posição 3 & 742,75 & 714,55 \\
\hline
\end{tabular}


fato oferece maior proteção balística quando comparada a uma superfície plana, pois pôde impedir a perfuração total do conjunto em alguns disparos. Entretanto, a região descendente da placa ondulada constitui uma região fragilizada, em que o desempenho em termos de proteção balística é comprometido.

Conforme observado por Jankowiak et al. [9], outra característica importante observada é que uma distância de espaçamento entre as placas torna a blindagem mais eficiente. Esse fato também pode explicar uma eventual queda de desempenho da blindagem após o primeiro impacto (na sequência I, 2, 3 e 4), pois a solda que ligava as duas placas e o epóxi usado para separação fraturaram, aproximando as placas.

\section{CONCLUSÃo}

Conforme observado nas simulações computacionais e nos ensaios balísticos, a configuração com base em placas onduladas de fato oferece maior proteção balística quando comparada à configuração com placas planas. Entretanto, como apresentado em ambas as análises, a região descendente da placa ondulada constitui uma região de menor proteção balística.

A região com variação angular foi responsável por defletir o movimento do projétil, embora não fosse possível avaliar o desvio da trajetória desse projétil.

Corroborou-se também que um espaçamento entre as placas de blindagem aumenta o desempenho em proteção balística.

\section{REFERÊNCIAS}

I Gomes, A.V. Comportamento balístico de alumina com adição de nióbia e variação da geometria do alvo [tese]. Rio de Janeiro: Instituto Militar de Engenharia; 2004.

2 Trindade, W. Influência da Geometria e da Microsestrutura no Comportamento Dinâmico da Alumina Aditivada com Nióbia [tese]. Rio de Janeiro: Instituto Militar de Engenharia; 2012.

3 Associação Brasileira de Normas Técnicas - ABNT. NBR 15000: Blindagens Para Impactos Balísticos — Classificação e Critérios de Avaliação. Rio de Janeiro: ABNT; 2005.

4 Rosenberg Z, Dekel E. Terminal ballistics. New York: Springer; 2012.

5 Johnson GR, Cook WH. A constitutive model and data for metals subjected to large strains, high strain rates, and high temperature. In: Proceedings of the 7th International Symposium on Ballistics; 1983 Apr 19-21; The Hague, Netherlands. The Netherlands: The Hague; 1983. p. 54I-554.

6 Steinberg DJ, Cochran SG, Guinan MW. A constitutive model for metals applicable at high-strain rate. Journal of Applied Physics. 1980;5I(3)

7 Society of Automotive Engineers - SAE. AMS65I2 - H: Steel, Maraging, Bars, Forgings, Tubing, and Rings I8Ni 7.8Co-4.9Mo - 0, IOAI Consumable Electrode Vacuum Melted, Annealed. New York: SAE; 2017.

8 United States Defense Standard. MIL-S-46850-D: Steel: Bar, Plate, Sheet, Strip, Forgings, and Extrusions, 18 Percent Nickel Alloy, Maraging, 200 ksi, 250 ksi, 300ksi, High Quality. Sterling: United States Defense Standard; 199 I.

9 Jankowiak T, Rusine A, Wood P. A numerical analysis of the dynamic behaviour of sheet steel perforated by a conical projectile under ballistic conditions. Finite Elements in Analysis and Design. 20I3;65:39-49.

Recebido em: 14 Jan, 2020

Aceito em: II Maio 2020 